\begin{tabular}{|c|c|}
\hline $\begin{array}{r}\text { PRAMANA } \\
- \text { journal of } \\
\text { physics }\end{array}$ & (c) Indian Academy of Sciences \\
\hline
\end{tabular}

\title{
The origin of the solar magnetic cycle
}

\author{
ARNAB RAI CHOUDHURI \\ Department of Physics, Indian Institute of Science, Bangalore 560 012, India \\ E-mail: arnab@physics.iisc.ernet.in
}

\begin{abstract}
After summarizing the relevant observational data, we discuss how a study of flux tube dynamics in the solar convection zone helps us to understand the formation of sunspots. Then we introduce the flux transport dynamo model and assess its success in modelling both the solar cycle and its departures from strictly periodic behaviour.
\end{abstract}

Keywords. Sun: activity; Sun: magnetic fields; sunspots.

PACS Nos 96.60.Hv; 96.60.Jw; 96.60.Q-; 96.60.qd

\section{Introduction}

It is a great honour for me to give a plenary talk in the Chandra Centenary Symposium. As a graduate student of Gene Parker in the early 1980s, I had the privilege of working for four years in an office about 4 or 5 doors down the corridor from Chandra's office. Those of you who had visited University of Chicago in those days may know that most of the astronomy faculty and students were in a building called Astronomy and Astrophysics Center. The building next to it - Laboratory for Astrophysics and Space Research - mainly housed the large cosmic ray research group. However, two of the most beautiful offices in that building were given to two theoretical astrophysicists - Chandra and Parker. Chandra stopped taking students after a heart attack in the 1970s and there were no students working with him when I was in Chicago. For a while I was the only theory student having a very nice office in that building. When I was attending the first AAS meeting of my life, somebody asked me at the dinner table, "How big is your theory group?" I replied: "It is a very small theory group with only three members." The next question was, "Who are the members?" I casually said: "Oh, besides myself, the other two members of our small theory group are Subrahmanyan Chandrasekhar and Eugene Parker."

There was such an aura around Chandra that, like most other graduate students, I was in awe and always tried my best to keep away from him. Since I was the only other Indian in the building, Chandra seemed somewhat curious about me and often asked Gene what I was doing. Gene used to tell me that I should overcome my fear of Chandra and should talk to him some time. A few days after my first paper dealing with the solar dynamo problem appeared [1], while walking along the corridor, I saw Chandra coming from the 


\section{Arnab Rai Choudhuri}

opposite direction. Normally we would walk past each other as if we were strangers. That day, to my utter consternation, Chandra suddenly stopped when he came close to me and looked straight into my eyes. Then he said: "I have seen your paper. It is a nice piece of work." Without giving me any time to recover from my dazed state or to respond, Chandra immediately walked away. I should mention that I had always been a great admirer of Chandra's style of writing. Although that first paper of mine presented a relatively unimportant calculation, it was deliberately written in imitation of the Chandra style. You may want to compare that paper [1] with the famous S. Candlestickmaker paper! I have a hope that, if Chandra were present here today, he would have taken some interest in the subject of my presentation.

All of you know about the 11-year periodicity of the sunspot cycle. There also seems to be a 50-year periodicity in this field which you may not be aware of! So let me begin by telling you about this 50 -year periodicity. It appears that major breakthroughs in this field take place approximately at the intervals of 50 years. (1) A little more than 150 years ago, the German amateur astronomer Schwabe [2] reported the first discovery of the sunspot cycle. (2) About 100 years ago, Hale [3] found the evidence of Zeeman splitting in the spectra of sunspots, thereby concluding that sunspots are regions of concentrated magnetic field. It may be mentioned that this was a momentous discovery in the history of physics because this was the first time somebody found a conclusive evidence of largescale magnetic fields outside the Earth's environment. Now we know that magnetic fields are ubiquitous in the astronomical Universe. With Hale's discovery, it also became clear that the sunspot cycle is essentially a magnetic cycle of the Sun. (3) About 50 years ago, Parker [4] finally formulated the turbulent dynamo theory, which still provides the starting point of our understanding of how magnetic fields arise in astronomical systems.

Even without a theoretical model of this 50-year periodicity, you should be able to make a simple extrapolation and predict that another major breakthrough in this field should be taking place right now. We are going to argue that such a breakthrough is indeed happening at the present time. Some of the earlier breakthroughs were achieved single-handedly by extraordinary individuals like Hale and Parker. Now we probably live in a less heroic age. The present breakthrough is a result of efforts due to many groups around the world, in which our group in Bangalore also has made some contributions.

\section{Some observational considerations}

Let us begin by looking at figure 1, which plots the sunspot number as a function of time from the year 1610. Galileo and some of his contemporaries were the first scientists to study sunspots systematically. The initial entries in figure 1 are based on their records. Then, for nearly a century, sunspots were rarely seen - a period known as the Maunder minimum. Afterwards the sunspot number has varied periodically with a rough period of about 11 years, although we see a considerable amount of irregularity. Some cycles are stronger than the average and some are weaker. An intriguing question is whether we can predict the strength of a cycle in advance. Simple methods like expanding the last few cycles in a Fourier series and continuing the series to predict the next cycles have failed completely in the past. It is clearly not a problem of merely extending a mathematical series and we presumably need a proper understanding of what causes the irregularities of the cycles if 
The origin of the solar magnetic cycle

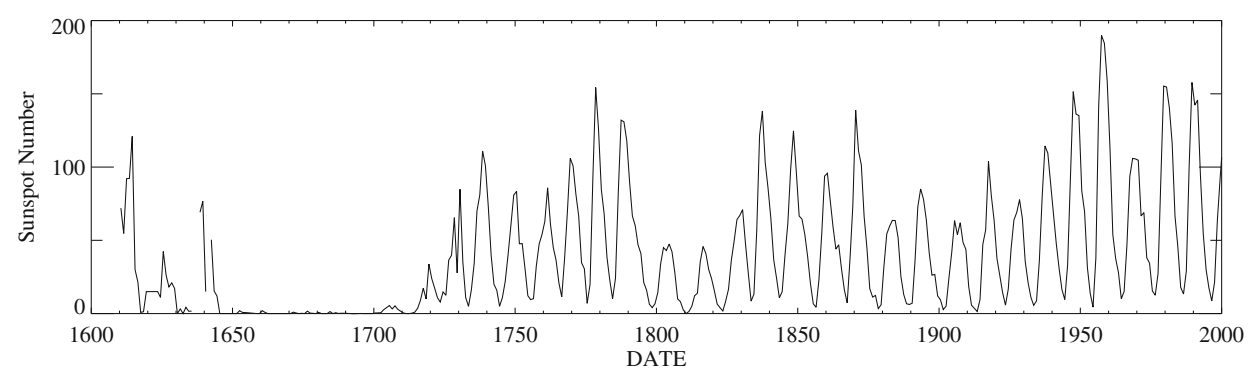

Figure 1. The yearly averaged number of sunspots plotted against time for the period 1610-2000.

we hope to predict a future cycle successfully. When we discuss the causes of irregularities in sunspot cycles in $\S 5$, we shall address the question whether our understanding of sunspot cycles at the present time is good enough to make such predictions.

A few years after Schwabe's discovery of the sunspot cycle [2], Carrington [5] noted that sunspots seemed to appear at lower and lower latitudes with the progress of the solar cycle. It may be mentioned that individual sunspots live from a few days to a few weeks. Most of the sunspots in the early phase of a solar cycle are seen between $30^{\circ}$ and $40^{\circ}$. As the cycle advances, new sunspots are found at increasingly lower latitudes. Then a fresh cycle begins with sunspots appearing again at high latitudes. Maunder [6] made the first graphical representation of this. In a time-latitude plot, the latitudes where sunspots were seen at a particular time can be marked by black bars. Figure 2 shows one such plot. The explanation of the grey-scale background will be provided later. The sunspot distribution in a time-latitude plot is often referred to as a butterfly diagram, since the pattern (the regions marked in black bars in figure 2) reminds one of butterflies.

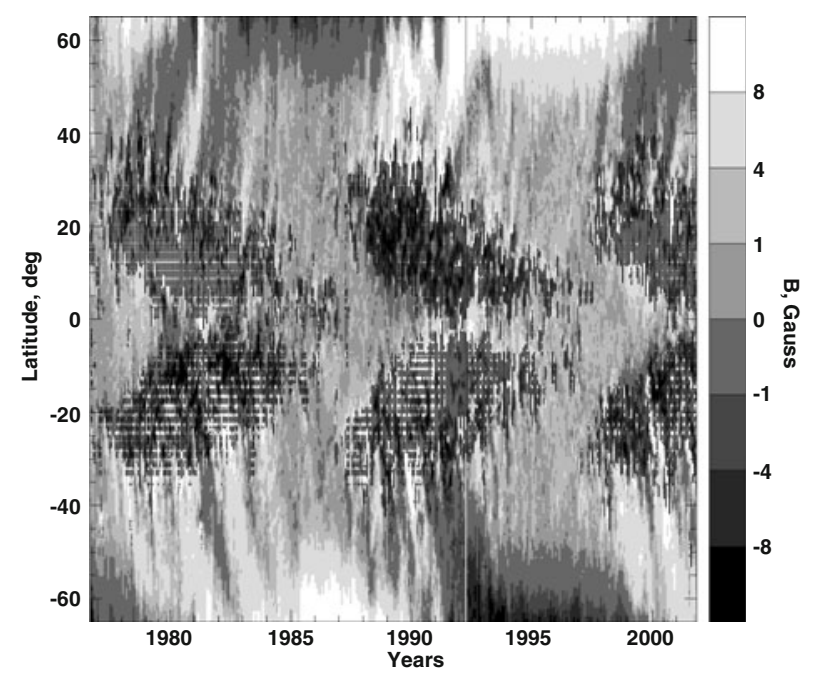

Figure 2. A 'butterfly diagram' of sunspots, with shades of grey showing the latitudetime distribution of longitudinally averaged weak, diffuse magnetic field ( $B$ is in Gauss). 


\section{Arnab Rai Choudhuri}

We have mentioned Hale's discovery of magnetic fields in sunspots [3]. A large sunspot has a typical magnetic field of about $3000 \mathrm{G}$. A few years later, Hale and his coworkers made another significant discovery [7]. Often two large sunspots are seen side by side. Hale et al [7] found that they invariably have opposite polarities. Figure 3 shows a magnetogram map of the Sun in which white and black indicate respectively regions of strong positive and negative polarities, grey being put in regions where the magnetic field is below a threshold. A bipolar sunspot pair appears as a white patch and a black patch side by side. You may note in figure 3 that the right sunspots in the sunspot pairs in the northern hemisphere are positive, whereas the right sunspots in the sunspot pairs in the southern hemisphere are negative. This is the case for a particular cycle. In the next cycle, the polarity reverses. The right sunspots in the northern hemisphere would become negative in the next cycle and the right sunspots in the southern hemisphere would become positive. If we only look at the sunspot number, we may think that the sunspot cycle has a period of 11 years. However, by taking account of the configuration of the magnetic field, we realize that the Sun's magnetic cycle has actually a period of 22 years.

You may note another thing in figure 3 . The line joining the centres of a bipolar sunspot pair is, on an average, nearly parallel to the solar equator. Hale's co-worker Joy, however, noted that there is a systematic tilt of this line with respect to the equator (the right sunspot in a pair appearing closer to the equator) and that this tilt increases with latitude [7]. This result is usually known as Joy's law. The tilts, however, show a considerable amount of scatter around the mean given by Joy's law. As we shall see later, this law of tilts of sunspot pairs plays a very important role in solar dynamo theory.

We shall present a detailed discussion in $\$ 3$ on how the bipolar sunspot pairs arise. For the time being, let us just mention that there has to be a strand of sub-surface magnetic field

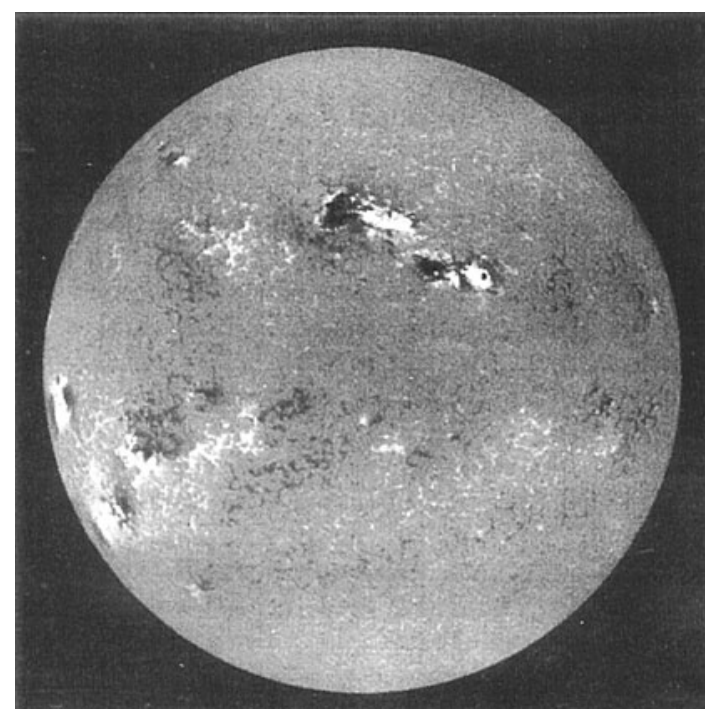

Figure 3. A magnetogram image of the full solar disk. The regions with positive and negative magnetic polarities are respectively shown in white and black, with grey indicating regions where the magnetic field is weak. 


\section{The origin of the solar magnetic cycle}

which occasionally breaks out of the solar surface as shown in figure $7 \mathrm{~b}$. Then magnetic field lines would come out of one sunspot (making its polarity positive) and would go down into the other sunspot (making its polarity negative). A look at figure 3 suggests that there must be a sub-surface magnetic field with field lines going from the right to the left in the northern hemisphere and there must be an oppositely directed magnetic field in the southern hemisphere. Such a magnetic field in the azimuthal direction is called a toroidal field. This seems to be the dominant component of the magnetic field in the Sun. In contrast, the magnetic field of the Earth seems to be of poloidal nature.

In his seminal paper on the turbulent dynamo, Parker [4] proposed that the sunspot cycle is produced by the oscillation between toroidal and poloidal components of the Sun's magnetic field, just as we see the oscillation between kinetic and potential energies in a simple harmonic oscillator. This was a truly extraordinary suggestion because almost nothing was known about the Sun's poloidal field at that time. Babcock and Babcock [8] were the first to detect the weak poloidal field having a strength of about $10 \mathrm{G}$ near the Sun's poles. Over the last few years, there is increasing evidence that the field outside the sunspots is actually not weak and diffuse, but concentrated in intermittent flux concentrations [9]. Only in low-resolution magnetograms in which these flux concentrations are not resolved, the field appears weak and diffuse. This seems to be the case even in the polar regions [10]. However, we shall not get into a more detailed discussion of this point here. Only from mid-1970s, we have systematic data of the Sun's polar fields. Figure 4 shows the polar fields of the Sun plotted as a function of time ( $\mathrm{N}$ and $\mathrm{S}$ indicating north and south poles), with the sunspot number plotted below. It is clear that the sunspot number, which is a
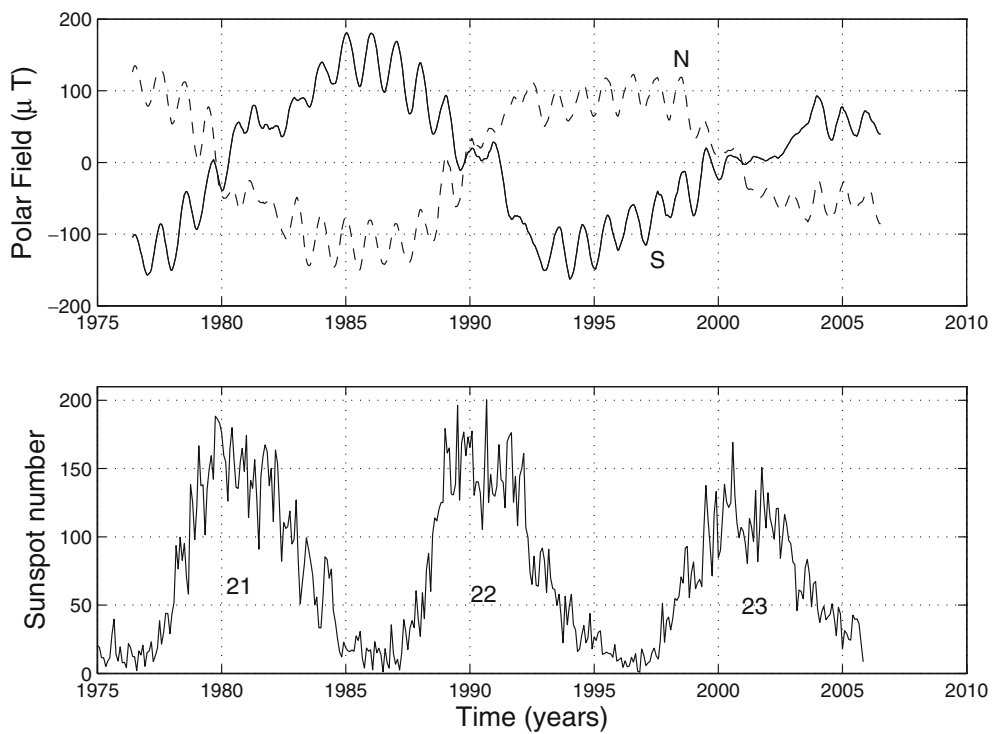

Figure 4. The polar field of the Sun as a function of time (on the basis of the Wilcox Solar Observatory data) with the sunspot number shown below. The top panel, with $\mathrm{N}$ and $\mathrm{S}$ representing north and south poles, is adapted from [51]. The yearly modulations in the measurements of the polar fields are due to the Sun's polar axis being slightly inclined to the orbital plane of the Earth's revolution around the Sun. 


\section{Arnab Rai Choudhuri}

proxy of the toroidal field, is maximum at a time when the polar field is nearly zero. On the other hand, the polar field is strongest when the sunspot number is nearly zero. This clearly shows an oscillation between the toroidal and poloidal components, as envisaged by Parker [4]. The theoretical reason behind this oscillation will be discussed in $\S 4$.

Let us make some more remarks on the poloidal field of the Sun. It was found that there were large unipolar patches of diffuse magnetic field on the solar surface which migrated poleward [11]. Even when averaged over longitude, one finds predominantly one polarity in a belt of latitude which drifts poleward [12]. The reversal of polar field, which occurs at the time of the sunspot maximum [13], presumably takes place when sufficient field of opposite polarity has been brought to the poles. Along with the butterfly diagram of sunspots, figure 2 also shows the distribution of the longitude-averaged poloidal field in a time-latitude plot. The various shades of grey indicate values of the longitude-averaged poloidal field. While the sunspots appear at lower and lower latitudes with the progress of the solar cycle, the poloidal field migrates poleward. The reason behind the poleward migration of the poloidal field is a meridional circulation in the Sun which involves a flow of gas at the surface from the equatorial region to the polar region, having an amplitude of about $20 \mathrm{~m} \mathrm{~s}^{-1}$ [14]. The poloidal field is carried poleward by this meridional circulation.

It may be noted that all the observations discussed above pertain to magnetic fields at the Sun's surface. We have no direct information about magnetic fields underneath the Sun's surface. In dynamo theory, we need to study the interactions between the magnetic fields and velocity fields. So let us now look at the nature of the velocity fields of the Sun.

Stellar structure models suggest that the energy produced by nuclear reactions at the centre of the Sun is transported outward by radiative transfer to a radius of about $0.7 R_{\odot}$ (where $R_{\odot}$ is the solar radius). However, from about $0.7 R_{\odot}$ to $R_{\odot}$, energy is transported by convection. This region is called the convection zone, within which the plasma is in a turbulent state with hot gas going up and cold gas coming down. The turbulent diffusivity of the convection zone is the main source of diffusion in the dynamo problem. We have already pointed out that there is a meridional circulation which is poleward near the solar surface at the top of the convection zone. This meridional circulation is supposed to be driven by the turbulent stresses in the convection zone, though our theoretical understanding of this subject is rather limited at the present time. It is generally believed that the meridional circulation at the bottom of the convection zone has to go from the polar region to the equatorial region to conserve mass, although we do not have a direct evidence for it yet. This meridional circulation plays a tremendously important role in current dynamo models, as we shall see later.

We finally come to what is probably the most important part of the Sun's velocity field for us - the differential rotation. Unlike the Earth which rotates like a solid body, the Sun has the angular velocity varying over it. It has been known for a long time that the angular velocity near the Sun's equator is faster than that at the Sun's polar regions. In the early years of dynamo research, theorists used to make various assumptions about the distribution of angular velocity in the Sun's interior. An amazing development of the last few decades has been helioseismology - the study of the oscillations of the Sun. These oscillations have allowed us to probe various properties of the solar interior. One of the most extraordinary outcomes of helioseismology is that solar physicists have been able to construct a map of angular velocity distribution in the interior of the Sun (see, for example, [15]). A version of this map is shown in figure 5. It is seen that there is strong differential 


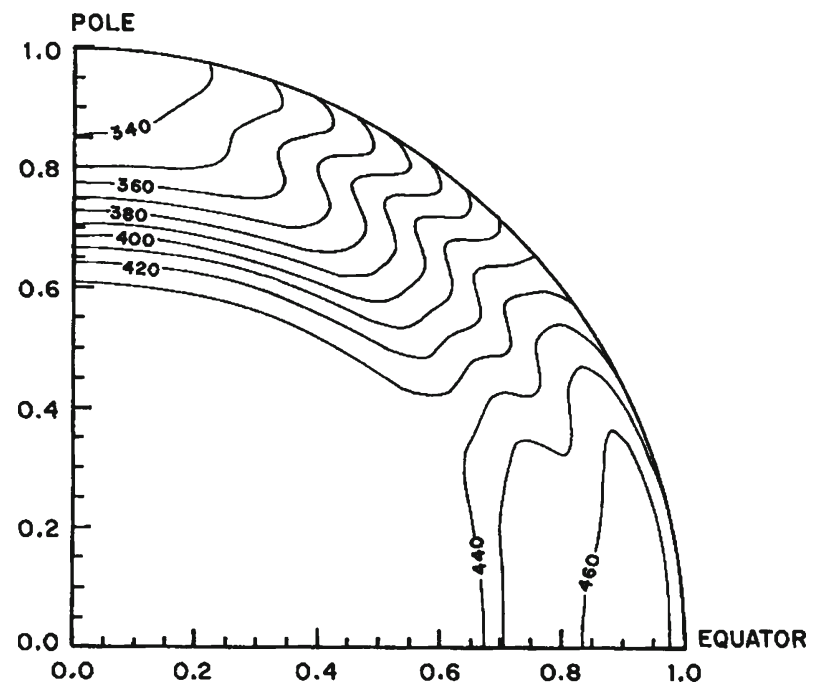

Figure 5. The contours of constant angular velocity inside the Sun, as obtained by helioseismology. The contours are marked with rotation frequency in $\mathrm{nHz}$. It may be noted that frequencies of $340 \mathrm{nHz}$ and $450 \mathrm{nHz}$ correspond respectively to rotation periods of 34 days and 25.7 days. Courtesy: J Christensen-Dalsgaard and M J Thomson.

rotation (i.e. a strong gradient of angular velocity) at the bottom of the solar convection zone. This relatively thin layer of concentrated differential rotation is called the tachocline.

We have now come to the end of our discussion of what we know about the magnetic and the velocity fields of the Sun. The aim of solar dynamo theory is the following. Given our knowledge of the velocity fields of the Sun, we need to study the interactions between the velocity and magnetic fields in the Sun's interior such that all the surface observations of magnetic fields are properly explained - a fairly daunting problem, of which the full solution is still a distant dream.

\section{Formation of sunspots}

All our theoretical considerations are based on magnetohydrodynamics (MHD). An introduction to its basic concepts can be found in Chs. 14-16 of [16] or Ch. 8 of [17]. Let us begin by mentioning some concepts of MHD which we shall be using repeatedly. We know that a magnetic field has a pressure $B^{2} / 2 \mu$ associated with it, along with a tension along the field lines. The other result which is going to be of central importance to us is the theorem due to Alfvén [18] which says that, when the magnetic Reynolds number is sufficiently high, magnetic fields are frozen in the plasma and get carried by the velocity fields of the plasma. Because of the high magnetic Reynolds number in the Sun, we expect this theorem to hold - at least approximately.

Since energy is transported by convection in the layers below the Sun's surface, sunspots are basically regions of concentrated magnetic field sitting in a convecting fluid. To understand why the magnetic field remains concentrated in structures like sunspots instead of spreading out more evenly, we need to study the interaction of the magnetic field with the 


\section{Arnab Rai Choudhuri}

convection in the plasma. This subject is known as magnetoconvection. The linear theory of convection in the presence of a vertical magnetic field was studied by Chandrasekhar [19]. The nonlinear evolution of the system, however, can only be found from numerical simulations pioneered by Weiss [20]. Since the tension of magnetic field lines opposes convection, it was found that space gets separated into two kinds of regions. In certain regions, magnetic field is excluded and vigorous convection takes place. In other regions, magnetic field gets concentrated, and the tension of magnetic field lines suppresses convection in those regions. Sunspots are presumably such regions where magnetic field is piled up by surrounding convection. Since heat transport is inhibited there due to the suppression of convection, sunspots look darker than the surrounding regions. Although we have no direct information about the state of the magnetic field under the Sun's surface, it is expected that the interactions with convection would keep the magnetic field concentrated in bundles of field lines throughout the solar convection zone. Such a concentrated bundle of magnetic field lines is called a flux tube.

One important consequence of Alfvén's theorem of flux freezing for the Sun is the following. If there is any poloidal field line going through the Sun, differential rotation will drag it out to produce a toroidal field, as shown in figure 6 . The production of the toroidal field is expected to be strongest in the tachocline at the bottom of the convection zone where the gradient of angular velocity is concentrated (see figure 5). Due to interactions with convection there, the toroidal field should exist in the form of horizontal flux tubes. If a part of such a flux tube rises up and pierces the solar surface as shown in figure $7 \mathrm{~b}$, then we expect to have two sunspots with opposite polarities at the same latitude. But how can a configuration like figure $7 \mathrm{~b}$ arise? The answer to this question was provided by Parker [21] through his idea of magnetic buoyancy. We need to have a pressure balance across the surface of a flux tube. Since the pressure of magnetic field inside the flux tube is $B^{2} / 2 \mu$, the interior pressure is a sum of this pressure and the gas pressure $p_{\text {in. }}$. On the other hand, the only pressure outside is the gas pressure $p_{\text {out }}$. Hence we must have

$$
p_{\text {out }}=p_{\text {in }}+\frac{B^{2}}{2 \mu}
$$

(a)

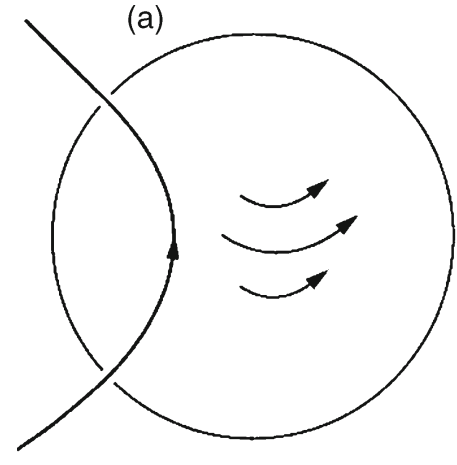

(b)

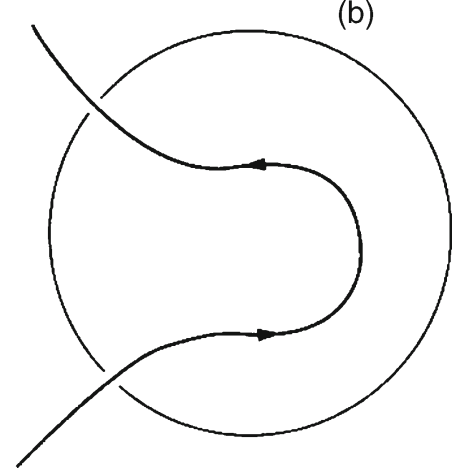

Figure 6. The production of a strong toroidal magnetic field underneath the Sun's surface. (a) An initial poloidal field line. (b) A sketch of the field line after it has been stretched by the faster rotation near the equatorial region. 
(a)
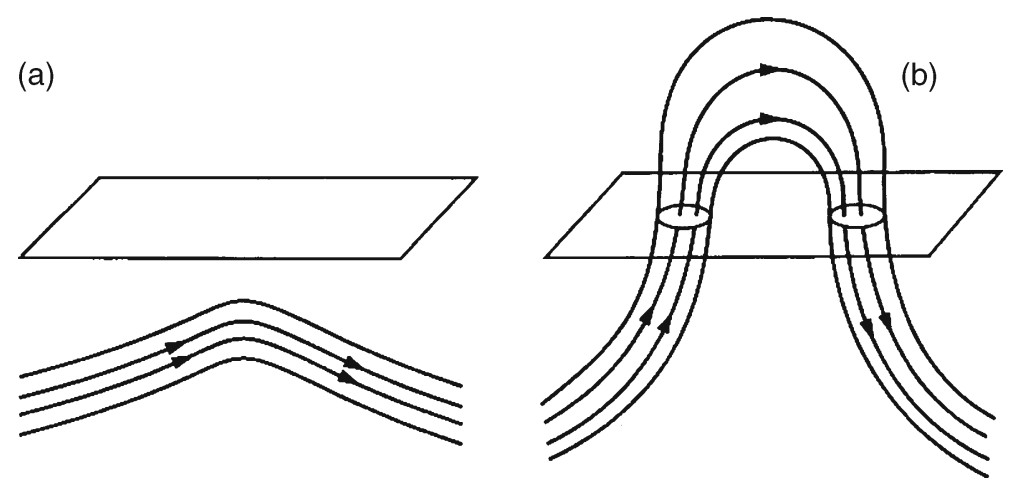

Figure 7. Magnetic buoyancy of a flux tube. (a) A nearly horizontal flux tube under the solar surface. (b) The flux tube after its upper part has risen through the solar surface.

to maintain pressure balance across the surface of a flux tube. It follows that

$$
p_{\text {in }} \leq p_{\text {out }},
$$

which often, though not always, implies that the density inside the flux tube is less than the surrounding density. If this happens in a part of the flux tube, then that part becomes buoyant and rises against the gravitational field to produce the configuration of figure $7 \mathrm{~b}$ starting from figure $7 \mathrm{a}$. It is seen in figure $6 \mathrm{~b}$ that the toroidal fields in the two hemispheres are in the opposite directions. If parts of these toroidal fields rise in the two hemispheres to produce the bipolar sunspot pairs, we have a natural explanation as to why the sunspot pairs should have the opposite polarity in the two hemispheres as seen in the magnetogram map of figure 3 .

It can be shown that magnetic buoyancy is particularly destabilizing in the interior of the convection zone, where convective instability and magnetic buoyancy reinforce each other. On the other hand, if a region is stable against convection, then magnetic buoyancy can be partially suppressed there (see, for example, $\$ 8.8$ in [22]). Since the toroidal flux tube is produced at the bottom of the convection zone, we may expect some parts of it to come into the convection zone and become buoyant, whereas other parts may remain underneath the bottom of the convection zone and stay anchored there due to the suppression of magnetic buoyancy. A part of the flux tube coming within the convection zone is expected to rise and eventually reach the solar surface to form sunspots, as given in figure 7. To model the formation of bipolar sunspots, we have to study the dynamics of flux tubes rising through the convection zone due to magnetic buoyancy.

The best way to study this problem is to treat it as an initial-value problem. First, an initial configuration with a magnetic flux ring at the bottom of the convection zone, having a part coming inside the convection zone, is specified, and then its subsequent evolution is studied numerically. The evolution depends on the strength of magnetic buoyancy, which is in turn determined by the value of the magnetic field. We shall give arguments in $\S 4$ about why most of the dynamo theorists till the early 1990s believed that the magnetic energy density should be in equipartition with the kinetic energy density of convection, i.e.

$$
\frac{B^{2}}{2 \mu} \approx \frac{1}{2} \rho v^{2}
$$




\section{Arnab Rai Choudhuri}

This suggests $B \approx 10^{4} \mathrm{G}$ on the basis of standard models of the convection zone. If we use full MHD equations to study the evolution of the flux tube, then the calculations become extremely complicated. However, if the radius of cross-section of the flux tube is smaller than the various scale heights, then it is possible to derive an equation for flux tube dynamics from the MHD equations [23,24]. Even this flux tube equation is a sufficiently complicated nonlinear equation and has to be solved numerically. The evolution of such magnetic flux tubes due to magnetic buoyancy (starting from the bottom of the convection zone) was studied by Choudhuri and Gilman [25] and Choudhuri [26]. It was found that the Coriolis force due to the Sun's rotation plays a much more important role in this problem than what anybody suspected before. If the initial magnetic field is taken to have a strength of around $10^{4} \mathrm{G}$ as suggested by (3), then the flux tubes move parallel to the rotation axis and emerge at very high latitudes rather than at latitudes where sunspots are seen. Only if the initial magnetic field is taken as strong as $10^{5} \mathrm{G}$, then the magnetic buoyancy is strong enough to overpower the Coriolis force and the magnetic flux tubes can rise radially to emerge at low latitudes.

D'Silva and Choudhuri [27] extended these calculations to look at the tilts of emerging bipolar regions at the surface. These tilts are also produced by the action of the Coriolis force on the rising flux tube. Figure 8 taken from [27] shows the observational tilt vs. latitude plot of bipolar sunspots (i.e. Joy's law) along with the theoretical plots obtained by assuming different values of the initial magnetic field. It is clearly seen that the theory fits observations only if the initial magnetic field is about $10^{5} \mathrm{G}$. If the magnetic field is much stronger, then the Coriolis force is unable to produce much tilt. On the other

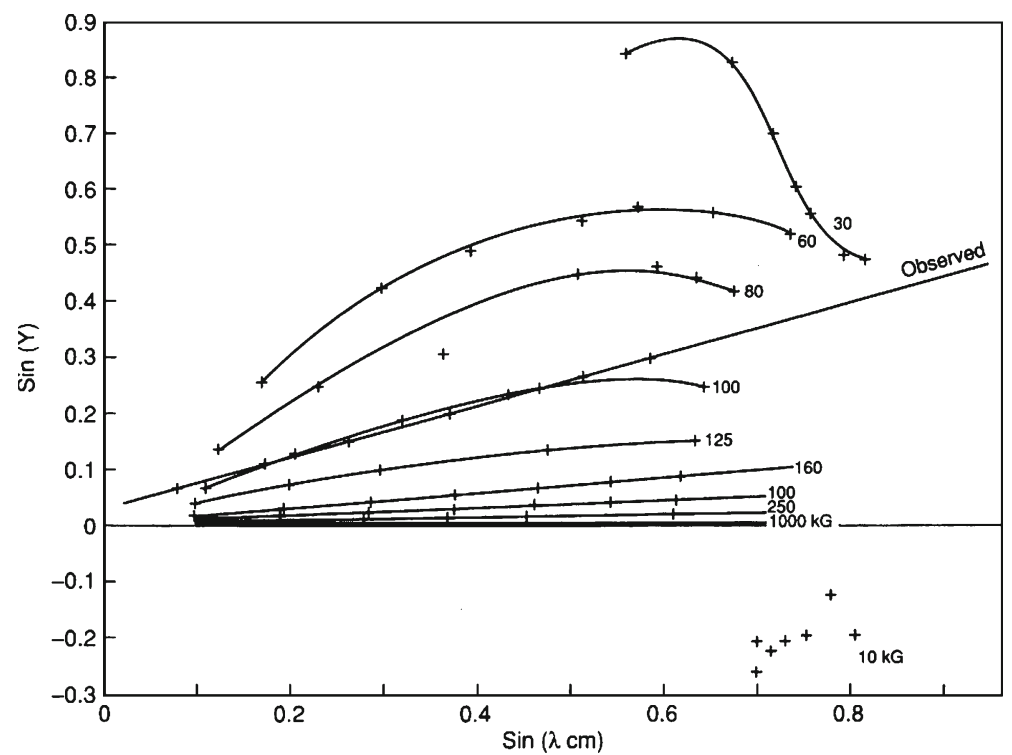

Figure 8. Plots of $\sin ($ tilt) against $\sin$ (latitude) theoretically obtained for different initial values of magnetic field indicated in $\mathrm{kG}$. The observational data indicated by the straight line fit the theoretical curve for initial magnetic field $100 \mathrm{kG}$ (i.e. $10^{5} \mathrm{G}$ ) (reproduced from D'Silva and Choudhuri [27]). 


\section{The origin of the solar magnetic cycle}

hand, flux tubes with weaker magnetic fields are diverted to high latitudes. Apart from providing the first quantitative explanation of Joy's law nearly three-quarters of a century after its discovery, D'Silva and Choudhuri [27] put the first stringent limit on the value of the toroidal magnetic field at the bottom of the convection zone. Several other authors $[28,29]$ soon performed similar calculations and confirmed the result. Initially some efforts were made to explore whether flux tubes with magnetic field given by (3) could satisfy various observational constraints by invoking extra effects [30,31]. However, the evidence kept mounting that the magnetic field at the bottom of the convection zone is indeed much stronger than the equipartition value given by (3).

We already mentioned that the tilts of active regions have a large amount of scatter around the mean given by Joy's law. In fact, it is found that active regions often emerge with initial tilts inconsistent with Joy's law and then the tilts change in the next few days to come closer to values given by Joy's law [32]. Longcope and Choudhuri [33] have argued that the vigorous convective turbulence in the upper layers of the convection zone exerts a random force on the tops of the rising flux loops, causing a scatter around Joy's law, and then the tilt of the flux tube relaxes to the appropriate value after the emergence of the top of the tube through the solar surface when the top is no longer kicked by convective turbulence.

\section{Modelling the cycles from flux transport dynamo}

If we begin by assuming the Sun to have a poloidal field as shown in figure 6a, we saw that various properties of sunspot pairs can be explained. The differential rotation would stretch this poloidal field to produce the toroidal field, the interaction with convection would lead to toroidal flux tubes and then magnetic buoyancy would make these flux tubes rise to produce the bipolar sunspots. However, if there is no mechanism to replenish the poloidal field, then it would decay away and ultimately the whole process outlined here would stop. We now turn to the question as to how the poloidal field is produced. We invoke a mechanism first proposed by Babcock [34] and Leighton [35]. The name of Leighton should be known to most physicists as the second author of the celebrated Feynman Lectures [36].

Let us now explain what this Babcock-Leighton mechanism is. We pointed out in $\$ 2$ that bipolar sunspots have tilts increasing with latitude, in accordance with Joy's law. Then we discussed in $\S 3$ how this law was explained by D'Silva and Choudhuri [27] by considering the action of the Coriolis force on rising flux tubes. Now, a typical sunspot lives for a few days and the magnetic field of the sunspot diffuses in the surrounding region by turbulent diffusion after its decay. When a tilted bipolar sunspot pair with the right spot nearer the equator and the left spot at a higher latitude decays, the polarity of the right sunspot gets more diffused in the lower latitudes and the polarity of the left sunspot gets more diffused in the higher latitudes. Take a look at figure 3 to visualize this process. This process essentially gives rise to a poloidal field at the solar surface. Since sunspots form from the toroidal field due to magnetic buoyancy, a tilted bipolar sunspot pair can be viewed as a conduit through which a part of the toroidal field ultimately gets transformed into the poloidal field. The tilted sunspot pair forms from the toroidal and we get the poloidal field after its decay. This is the basic idea of poloidal field generation proposed by Babcock [34] and Leighton [35]. 


\section{Arnab Rai Choudhuri}

It may be noted that this Babcock-Leighton mechanism is somewhat different from the original proposal of Parker [4], which was elaborated further by Steenbeck et al [37]. According to this original proposal, the turbulence in the convection zone would involve helical motions due to the Coriolis force and the toroidal field would be twisted by this helical turbulence to produce the poloidal field. However, this process, often known as the $\alpha$-effect, can occur only if the maximum value of the toroidal magnetic field is such that the magnetic energy density does not exceed the kinetic energy of turbulence, as indicated by (3). As we already pointed out, flux tube simulations for modelling sunspot formation suggest that the toroidal field is about one order of magnitude stronger (about $10^{5} \mathrm{G}$ ) compared to what we get from (3) (about $10^{4} \mathrm{G}$ ). If the toroidal field is so strong, then the $\alpha$-effect as originally envisaged by Parker [4] cannot work and the Babcock-Leighton mechanism seems to be the likely mechanism by which the poloidal field is produced.

Figure 9 is a cartoon encapsulating how the solar dynamo operates. If you understand this cartoon, then you would have got the central point of this paper! The toroidal field is produced in the tachocline by the differential rotation stretching out the poloidal field. Then this toroidal field rises due to magnetic buoyancy to produce bipolar sunspots at the solar surface, where the poloidal field is generated by the Babcock-Leighton mechanism from these bipolar sunspots. The poloidal field so generated is carried by the meridional circulation first to the polar region and then underneath the surface to the tachocline to be stretched by the differential rotation, thus completing the cycle. The likely streamlines of meridional circulation are indicated in figure 9. This type of dynamo model in which the meridional circulation plays a crucial role is called a flux transport dynamo.

Most of the present day dynamo theorists believe that the solar dynamo operates in this way. Wang et al [38] proposed the idea of the flux transport dynamo. Choudhuri et al [39] and Durney [40] were the first to construct two-dimensional models of the flux transport

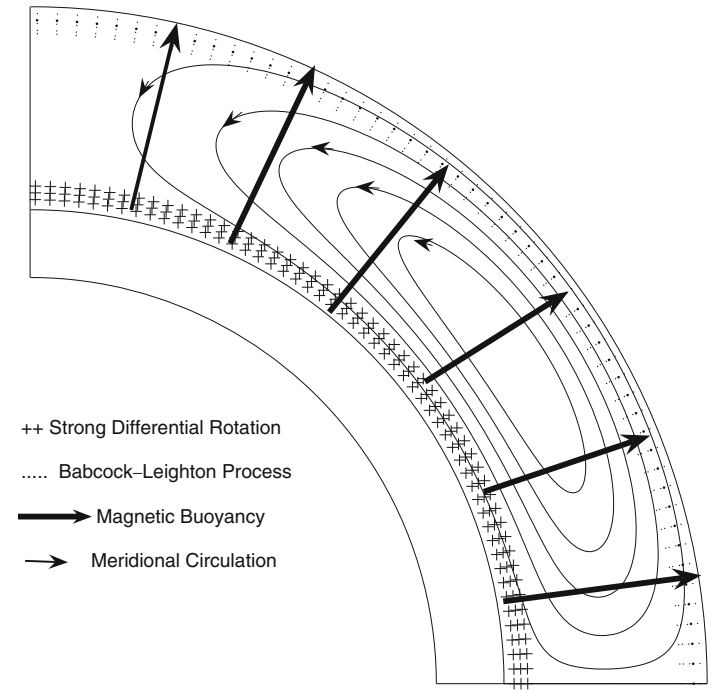

Figure 9. A cartoon explaining how the solar dynamo works within the convection zone. 


\section{The origin of the solar magnetic cycle}

dynamo to demonstrate that such a dynamo really does work. Initially it was thought that this type of dynamo model would not work due to a technical reason. There is a rule, known as the Parker-Yoshimura sign rule [4,41], which suggests that the type of dynamo outlined in figure 9 would produce a poleward dynamo wave. In other words, it was feared that such a theoretical model would suggest that sunspots should appear at higher and higher latitudes with the progress of the sunspot cycle rather than at lower and lower latitudes. Choudhuri et al [39] solved this puzzle by demonstrating that a sufficiently strong meridional circulation can override the Parker-Yoshimura sign rule and make the dynamo wave propagate equatorward. This paved the way for the subsequent growth of the flux transport dynamo model.

So far in this paper I have avoided getting into equations. For those who wish to see the equations, I now show the central equations of the flux transport dynamo theory. In spherical coordinates, we write the magnetic field as

$$
\mathbf{B}=B(r, \theta) \mathbf{e}_{\phi}+\nabla \times\left[A(r, \theta) \mathbf{e}_{\phi}\right],
$$

where $B(r, \theta)$ is the toroidal component and $A(r, \theta)$ gives the poloidal component. We can write the velocity field as $\mathbf{v}+r \sin \theta \Omega(r, \theta) \mathbf{e}_{\phi}$, where $\Omega(r, \theta)$ is the angular velocity in the interior of the Sun and $\mathbf{v}$ is the velocity of meridional circulation having components in $r$ and $\theta$ directions. Then the main equations telling us how the poloidal and the toroidal fields evolve with time are

$$
\begin{aligned}
& \frac{\partial A}{\partial t}+\frac{1}{s}(\mathbf{v} \cdot \nabla)(s A)=\lambda_{T}\left(\nabla^{2}-\frac{1}{s^{2}}\right) A+\alpha B, \\
& \frac{\partial B}{\partial t}+\frac{1}{r}\left[\frac{\partial}{\partial r}\left(r v_{r} B\right)+\frac{\partial}{\partial \theta}\left(v_{\theta} B\right)\right] \\
& =\lambda_{\mathrm{T}}\left(\nabla^{2}-\frac{1}{s^{2}}\right) B+s\left(\mathbf{B}_{p} \cdot \nabla\right) \Omega+\frac{1}{r} \frac{\mathrm{d} \lambda_{\mathrm{T}}}{\mathrm{d} r} \frac{\partial}{\partial r}(r B),
\end{aligned}
$$

where $s=r \sin \theta$ and $\lambda_{\mathrm{T}}$ is the turbulent diffusivity inside the convection zone. We should point out that eqs (5) and (6) are the mean field equations obtained by averaging over the turbulence in the convection zone and describe the mean behaviour of the average magnetic field. Since eqs (5) and (6) are coupled partial differential equations, nothing much can be done analytically. Our research group in IISc Bangalore has developed a numerical code Surya for studying the flux transport dynamo problem by solving eqs (5) and (6). I can send the code Surya and a detailed guide for using it to anybody who sends a request to my e-mail address arnab@physics.iisc.ernet.in.

Some of the first results obtained with Surya were presented by Nandy and Choudhuri [42] and Chatterjee et al [43]. I may mention that a modified version of Surya has even been used to study the evolution of magnetic fields in neutron stars [44,45]. Figure 10 shows a theoretical butterfly diagram of sunspots, superposed on contours in the time-latitude plot of the poloidal field on the solar surface. This theoretical figure obtained by the code Surya has to be compared with the corresponding observational figure given in figure 2. Given the fact that this was one of the first efforts of reproducing this observational figure from a theoretical model, hopefully most readers will agree that the match between theory and observations is not too bad. 
Arnab Rai Choudhuri

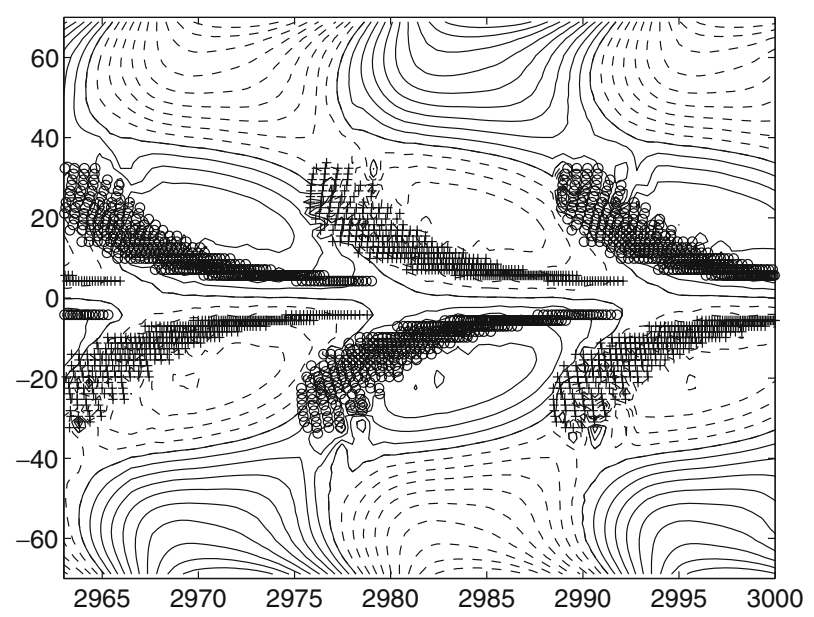

Figure 10. A theoretical butterfly diagram of sunspots superposed on contours of constant $B_{r}$ at the solar surface in a time-latitude plot. This figure is taken from Chatterjee et al [43].

The original flux transport dynamo model of Choudhuri, Schüssler and Dikpati [39] was developed at a time when Mausumi Dikpati was my PhD student. Afterwards she went to work in HAO Boulder and the parent model led to two offsprings: a high-diffusivity model and a low-diffusivity model. The diffusion times across the convection zone in these two models are of the order of 5 years and 200 years respectively. The high-diffusivity model has been developed in IISc Bangalore by me and my successive PhD students (Choudhuri, Nandy, Chatterjee, Jiang, Karak), whereas the low-diffusivity model has been developed by Dikpati and her co-workers in HAO (Dikpati, Charbonneau, Gilman, de Toma). The differences between these models have been systematically studied by Jiang et al [46] and Yeates et al [47]. Both these models are capable of producing oscillatory solutions resembling solar cycles. However, when we try to study the variabilities of the cycles, the two models give completely different results. We need to introduce fluctuations to cause variabilities in the cycles. In the high-diffusivity model, fluctuations spread all over the convection zone in about five years. On the other hand, in the low-diffusivity model, fluctuations essentially remain frozen during the cycle period. Thus the behaviours of the two models are totally different on introducing fluctuations. It may be mentioned that simple mixing length arguments suggest a reasonably high turbulent diffusivity (see p. 629 of [22]) consistent with what is used in the high-diffusivity model of the IISc Bangalore group.

\section{Irregularities of solar cycles and prospects for predicting future cycles}

Before coming to the question of what causes the irregularities of solar cycles, we take another look at the plot of polar fields in figure 4 . The polar field at the end of cycle 22 was weaker than the polar field in the previous sunspot minimum. We see that this weaker polar field was followed by the cycle 23 which was weaker than the previous cycle. Does this mean that there is a correlation between the polar field during a sunspot minimum and 
The origin of the solar magnetic cycle
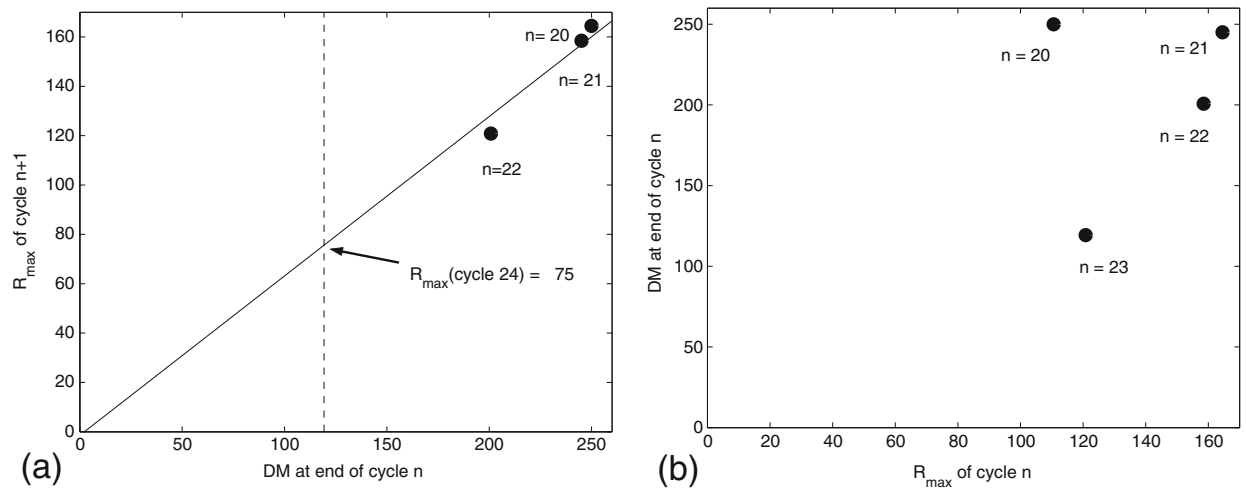

Figure 11. (a) shows a plot of the strength of cycle $n+1$ against the polar field at the end of cycle $n$. (b) shows a plot of the polar field at the end of cycle $n$ against the strength of the cycle $n$ (from Choudhuri [49]).

the next sunspot cycle? In figure 11a, we plot the polar field in the sunspot minimum along the horizontal axis and the strength of the next cycle along the vertical axis. Although there are only three data points so far, they lie so close to a straight line that one is tempted to conclude that there is a real correlation. There is a joke that astrophysicists often do statistics with one data point, whereas here we have three! On the other hand, figure 11b, which has the cycle strength along the horizontal axis and the polar field at the end of that cycle along the vertical axis, has points which are scattered around. Choudhuri et al [48] proposed the following to explain these observations. While an oscillation between toroidal and poloidal components takes place, the system gets random kicks at the epochs indicated in figure 12. Then the poloidal field and the next toroidal field should be correlated, as suggested by figure 11a. On the other hand, the random kick ensures that the toroidal field is not strongly correlated with the poloidal field coming after it, as seen in figure $11 \mathrm{~b}$.

If there is really a correlation between the polar field at the sunspot minimum and the next cycle, then one can use the polar field to predict the strength of the next cycle [50]. Since the polar field in the just concluded minimum has been rather weak (as seen in figure 4), several authors $[51,52]$ suggested that the coming cycle 24 will be rather weak. Very surprisingly, the first theoretical prediction based on a dynamo model made by Dikpati and Gilman [53] is that the cycle 24 will be very strong. Dikpati and Gilman [53] assumed the generation of the poloidal field from the toroidal field to be deterministic, which is not supported by observational data shown in figure 11b. Tobias et al [54] make the following comment on this work: "Any predictions made with such models should be treated with extreme caution (or perhaps disregarded), as they lack solid physical underpinnings." While we also

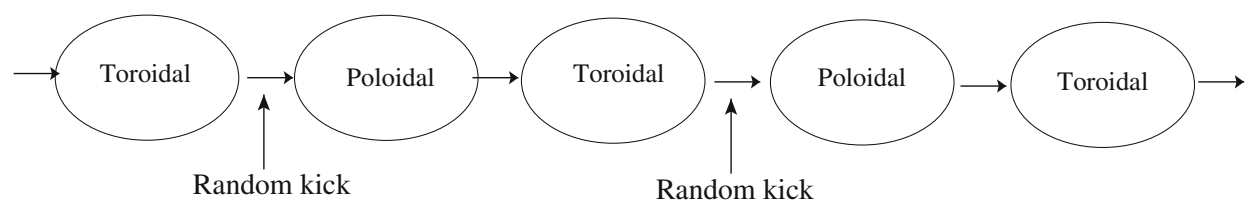

Figure 12. A schematic cartoon of the oscillation between toroidal and poloidal components, indicating the epochs when the system is subjected to random kicks. 


\section{Arnab Rai Choudhuri}

consider many aspects of the Dikpati-Gilman work wrong which will become apparent to the reader soon, we cannot also accept the opposite extreme viewpoint of Tobias et al [54], who suggested that the solar dynamo is a nonlinear chaotic system and predictions are impossible or useless. If that were the case, then we are left with no explanation for the correlation seen in figure 11a.

Let us now finally come to the theoretical question as to what produces the variabilities of cycles and whether we can predict the strength of a cycle before its advent. Some processes in nature can be predicted and some not. We can easily calculate the trajectory of a projectile by using elementary mechanics. On the other hand, when a dice is thrown, we cannot predict which side of the dice will face upward when it falls. Is the solar dynamo more like the trajectory of a projectile or more like the throw of a dice? Our point of view is that the solar dynamo is not a simple unified process, but a complex combination of several processes, some of which are predictable and the others are not. Let us look at the processes which make up the solar dynamo.

The flux transport dynamo model combines three basic processes. (i) The strong toroidal field is produced by the stretching of the poloidal field by differential rotation in the tachocline. (ii) The toroidal field generated in the tachocline gives rise to sunspots due to magnetic buoyancy and then the decay of tilted bipolar sunspots produces the poloidal field by the Babcock-Leighton mechanism. (iii) The poloidal field is advected by the meridional circulation first to high latitudes and then down to the tachocline, while diffusing as well. We believe that the processes (i) and (iii) are reasonably ordered and deterministic. In contrast, the process (ii) involves an element of randomness due to the following reason. The poloidal field produced from the decay of a tilted bipolar region by the Babcock-Leighton process depends on the tilt. While the average tilt of bipolar regions at a certain latitude is given by Joy's law, we observationally find quite a large scatter around this average. As we already pointed out, the action of the Coriolis force on the rising flux tubes gives rise to Joy's law [27], whereas convective buffeting of the rising flux tubes in the upper layers of the convection zone causes the scatter of the tilt angles [33]. This scatter in the tilt angles certainly introduces a randomness in the generation process of the poloidal field from the toroidal field. Choudhuri et al [48] identified it as the main source of irregularity in the dynamo process, which is in agreement with figure 12. It may be noted that Choudhuri [55] was the first to suggest several years ago that the randomness in the poloidal field generation process is the source of fluctuations in the dynamo.

The poloidal field gets built up during the declining phase of the cycle and becomes concentrated near the poles during the sunspot minimum. The polar field at the sunspot minimum produced in a theoretical mean field dynamo model is some kind of 'average' polar field during a typical sunspot minimum. The observed polar field during a particular sunspot minimum may be stronger or weaker than this average field. The theoretical dynamo model has to be updated by feeding the information of the observed polar field in an appropriate way, in order to model actual cycles. Choudhuri et al [48] proposed to model this in the following way. They ran the dynamo code from a minimum to the next minimum in the usual way. After stopping the code at the minimum, the poloidal field of the theoretical model was multiplied by a constant factor everywhere above $0.8 R_{\odot}$ to bring it in agreement with the observed poloidal field. Since some of the poloidal field at the bottom of the convection zone may have been produced in the still earlier cycles, it is left unchanged by not doing any updating below $0.8 R_{\odot}$. Only the poloidal field produced in 


\section{The origin of the solar magnetic cycle}

the last cycle which is concentrated in the upper layers gets updated. After this updating which takes care of the random kick shown in figure 12, we run the code till the next minimum, when the code is again stopped and the same procedure is repeated. Our solutions are now no longer self-generated solutions from a theoretical model alone, but are solutions in which the random aspect of the dynamo process has been corrected by feeding the observational data of polar fields into the theoretical model.

Before presenting the results obtained with this procedure, we come to the question as to how the correlation between the polar field at the sunspot minimum and the strength of the next cycle as seen in figure 11a may arise. This was first explained by Jiang et al [46]. The Babcock-Leighton process would first produce the poloidal field around the region $\mathrm{C}$ in figure 13. Then this poloidal field will be advected to the polar region $\mathrm{P}$ by meridional circulation and will also diffuse to the tachocline T. In the high-diffusivity model, this diffusion will take only about five years and the toroidal field of the next cycle will be produced from the poloidal field that has diffused to $\mathrm{T}$. If the poloidal field produced at $\mathrm{C}$ is strong, then both the polar field at $\mathrm{P}$ at the end of the cycle and the toroidal field at $\mathrm{T}$ for the next cycle will be strong (and vice versa). We thus see that the polar field at the end of a cycle and the strength of the next cycle will be correlated in the high-diffusivity model. But this will not happen in the low-diffusivity model where it will take more than 100 years for the poloidal field to diffuse from $\mathrm{C}$ to $\mathrm{T}$ and the poloidal field reaches the tachocline only due to the advection by meridional circulation taking a time of about 20 years. If we believe that the three data points in figure 11a indicate a real correlation, then we have to accept the high-diffusivity model!

Finally the solid line in figure 14 shows the sunspot number calculated from our highdiffusivity model [48]. Since systematic polar field measurements are available only from the mid-1970s, the procedure outlined above could be applied only from that time. It is seen from figure 14 that our model matches the last three cycles (dashed line) reasonably well

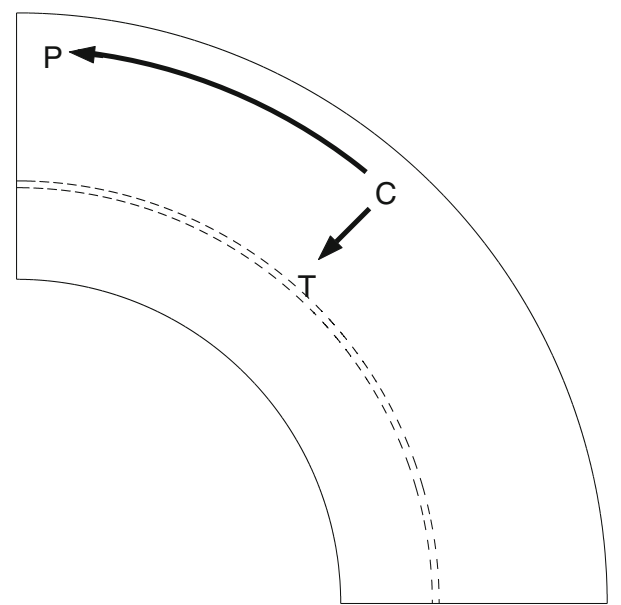

Figure 13. A sketch indicating how the poloidal field produced at $\mathrm{C}$ during a maximum gives rise to the polar field at $\mathrm{P}$ during the following sunspot minimum and the toroidal field at $\mathrm{T}$ during the next sunspot maximum (from [46]). 


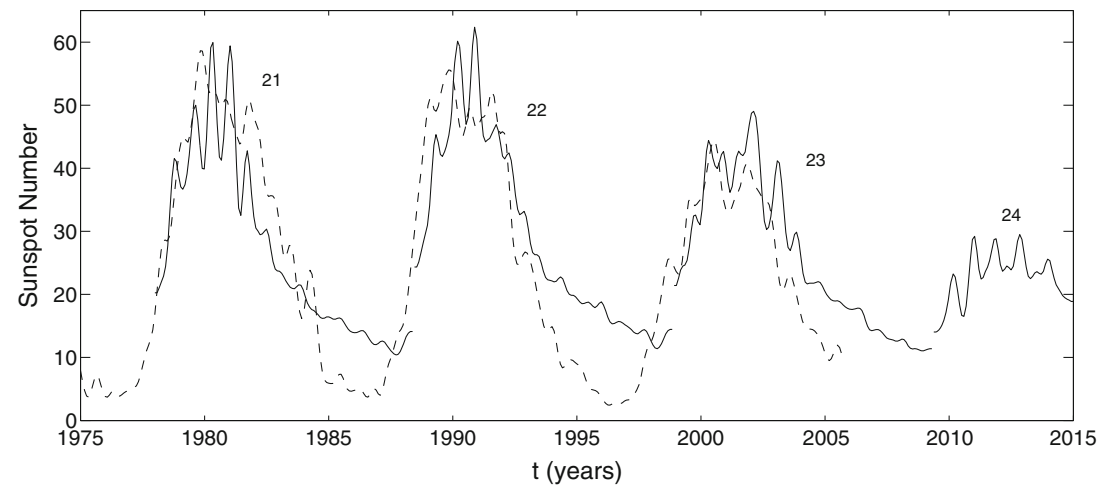

Figure 14. The theoretical monthly sunspot number (solid line) for the last few years as well as the upcoming next cycle, plotted along with the observational data (dashed line) for the last few years (from Choudhuri et al [48]).

and predicts a weak cycle 24 . It should be stressed that this is an inevitable consequence of the high-diffusivity model in which the strength of the cycle is correlated with the polar field in the previous sunspot minimum and we have fed the information in our calculation that the polar field in the just-concluded minimum was weak. We now wait for the Sungod to give a verdict on this prediction within a couple of years. It may be mentioned that over the last few years several authors [43,46,56-59] have given several independent arguments in support of the high-diffusivity model. If the next cycle 24 turns out to be weak (for which there are already enough indications), then that will further support the high-diffusivity model.

One important related question is whether our dynamo model can explain occurrences of extreme events like the Maunder minimum in the seventeenth century. Choudhuri and Karak [60] showed that the flux transport dynamo model can reproduce the Maunder minimum if we introduce a set of assumptions in the theoretical model. Whether this set of assumptions necessary for producing the Maunder minimum is justified on statistical grounds is an important question which needs to be investigated.

While the fluctuations in the Babcock-Leighton process seem to be the main source of irregularities in the sunspot cycle, the meridional circulation also has fluctuations and it has become apparent in the last few years that the fluctuations in meridional circulation also introduces irregularities in sunspot cycles [61]. Since this topic has started being studied systematically only recently [62-64], it would be premature to provide its summary here. It seems that the nonlinear aspects of the equations can also play important roles and there are some indications that the solar dynamo may be close to a point of chaotic bifurcation $[65,66]$. We are certainly far from a full theoretical understanding of the irregularities of the sunspot cycle.

\section{References}

[1] A R Choudhuri, Astrophys. J. 281, 846 (1984)

[2] S H Schwabe, Astron. Nachr. 21, 233 (1844) 
The origin of the solar magnetic cycle

[3] G E Hale, Astrophys. J. 28, 315 (1908)

[4] E N Parker, Astrophys. J. 122, 293 (1955)

[5] R C Carrington, Mon. Not. R. Astron. Soc. 19, 1 (1858)

[6] E W Maunder, Mon. Not. R. Astron. Soc. 64, 747 (1904)

[7] G E Hale, F Ellerman, S B Nicholson and A H Joy, Astrophys. J. 49, 153 (1919)

[8] H W Babcock and H D Babcock, Astrophys. J. 121, 349 (1955)

[9] J O Stenflo, Solar Phys. 32, 41 (1973)

[10] S Tsuneta et al, Astrophys. J. 688, 1374 (2008)

[11] V Bumba and R Howard, Astrophys. J. 141, 1502 (1965)

[12] Y-M Wang, A G Nash and N R Sheeley, Astrophys. J. 347, 529 (1989)

[13] H D Babcock, Astrophys. J. 130, 364 (1959)

[14] D H Hathaway and L Rightmire, Science 327, 1350 (2010)

[15] J Schou et al, Astrophys. J. 505, 390 (1998)

[16] A R Choudhuri, The physics of fluids and plasmas: An introduction for astrophysicists (Cambridge University Press, UK, 1998)

[17] A R Choudhuri, Astrophysics for physicists (Cambridge University Press, UK, 2010)

[18] H Alfvén, Ark. f. Mat. Astr. o. Fysik 29B, No. 2 (1942)

[19] S Chandrasekhar, Phil. Mag. 43, 501 (1952)

[20] N O Weiss, J. Fluid Mech. 108, 247 (1981)

[21] E N Parker, Astrophys. J. 121, 491 (1955)

[22] E N Parker, Cosmical magnetic fields (Oxford University Press, USA, 1979)

[23] H C Spruit, Astron. Astrophys. 98, 155 (1981)

[24] A R Choudhuri, Astron. Astrophys. 239, 335 (1990)

[25] A R Choudhuri and P A Gilman, Astrophys. J. 316, 788 (1987)

[26] A R Choudhuri, Solar Phys. 123, 217 (1989)

[27] S D'Silva and A R Choudhuri, Astron. Astrophys. 272, 621 (1993)

[28] Y Fan, G H Fisher and E E DeLuca, Astrophys. J. 405, 390 (1993)

[29] P Caligari, F Moreno-Insertis and M Schüssler, Astrophys. J. 441, 886 (1995)

[30] A R Choudhuri and S D'Silva, Astron. Astrophys. 239, 326 (1990)

[31] S Z D'Silva and A R Choudhuri, Solar Phys. 136, 201 (1991)

[32] R F Howard, Solar Phys. 169, 293 (1996)

[33] D Longcope and A R Choudhuri, Solar Phys. 205, 63 (2002)

[34] H W Babcock, Astrophys. J. 133, 572 (1961)

[35] R B Leighton, Astrophys. J. 156, 1 (1969)

[36] R P Feynman, R B Leighton and M Sands, The Feynman lectures on physics (Addison-Wesley, USA, 1963)

[37] M Steenbeck, F Krause and K-H Rädler, Z. Naturforsch. 21a, 1285 (1966)

[38] Y-M Wang, N R Sheeley and A G Nash, Astrophys. J. 383, 431 (1991)

[39] A R Choudhuri, M Schüssler and M Dikpati, Astron. Astrophys. 303, L29 (1995)

[40] B R Durney, Solar Phys. 160, 213 (1995)

[41] H Yoshimura, Astrophys. J. 201, 740 (1975)

[42] D Nandy and A R Choudhuri, Science 296, 1671 (2002)

[43] P Chatterjee, D Nandy and A R Choudhuri, Astron. Astrophys. 427, 1019 (2004)

[44] A R Choudhuri and S Konar, Mon. Not. R. Astron. Soc. 332, 933 (2002)

[45] S Konar and A R Choudhuri, Mon. Not. R. Astron. Soc. 348, 661 (2004)

[46] J Jiang, P Chatterjee and A R Choudhuri, Mon. Not. R. Astron. Soc. 381, 1527 (2007)

[47] A R Yeates, D Nandy and D H Mackay, Astrophys. J. 673, 544 (2008)

[48] A R Choudhuri, P Chatterjee and J Jiang, Phys. Rev. Lett. 98, 131103 (2007)

[49] A R Choudhuri, J. Astrophys. Astron. 29, 41 (2008)

[50] K H Schatten, P H Scherrer, L Svalgaard and J M Wilcox, Geo. Res. Lett. 5, 411 (1978) 
[51] L Svalgaard, E W Cliver and Y Kamide, Geo. Res. Lett. 32, L01104 (2005)

[52] K Schatten, Geo. Res. Lett. 32, L21106 (2005)

[53] M Dikpati and P A Gilman, Astrophys. J. 649, 498 (2006)

[54] S Tobias, D Hughes and N Weiss, Nature 442, 26 (2006)

[55] A R Choudhuri, Astron. Astrophys. 253, 277 (1992)

[56] P Chatterjee and A R Choudhuri, Solar Phys. 239, 29 (2006)

[57] A Goel and A R Choudhuri, Res. Astron. Astrophys. 9, 115 (2009)

[58] H Hotta and T Yokoyama, Astrophys. J. 709, 1009 (2010)

[59] H Hotta and T Yokoyama, Astrophys. J. 714, L308 (2010)

[60] A R Choudhuri and B B Karak, Res. Astron. Astrophys. 9, 953 (2009)

[61] P Charbonneau and M Dikpati, Astrophys. J. 543, 1027 (2000)

[62] B B Karak, Astrophys. J. 724, 1021 (2010)

[63] B B Karak and A R Choudhuri, Mon. Not. R. Astron. Soc. 410, 1503 (2011)

[64] D Nandy, A Muñoz-Jaramillo and P C H Martens, Nature 471, 80 (2011)

[65] P Charbonneau, C St-Jean and P Zacharias, Astrophys. J. 619, 613 (2005)

[66] P Charbonneau, G Beaubien and C St-Jean, Astrophys. J. 658, 657 (2007) 